\title{
Microstructural Characterization of Ion Implantation Effects in Aluminum Nitride Substrates for Energy-Scavenging Applications
}

\author{
F.S. Alleyne, R. Gronsky \\ Materials Science \& Engineering, University of California, Berkeley, Berkeley, CA 94720 USA
}

Due to increasing demands of energy consumption there is a dire need to bring energy storage and energy sources protocols to commercialization. One of the most attractive energy sources has roots in the popular recycling themes of other green technologies, commonly referred to energy scavenging. In its most promising conformation, energy scavenging converts cyclic mechanical vibrations in the environment or random mechanical pressure pulses, caused by sources ranging from operating machinery to human footfalls, into electrical energy via piezoelectric ${ }^{1}$ transducers. The most favorable material known for its combination of superior properties is lead zirconate titante, also known as $\mathrm{PZT}^{2}$. However, due to the presence of lead in these ceramic compounds there is much resistance to their application in anything "green" as lead based materials raises potential health implications during their manufacturing, recycling, or in-service applications. Therefore the search for alternative lead-free piezoelectrics has been underway for several years ${ }^{3}$ generating interest in aluminum nitride $(\mathrm{AlN})^{4}$, zinc oxide $(\mathrm{ZnO})^{4}$, and more recently polymeric materials ${ }^{5}$. In this investigation we have pursued the application of AlN as a lead-free piezoelectric candidate for energy-scavenging applications and exploit its compatibility with complementary-metal-oxide semiconductor (CMOS) manufacturing.

The processing pathways for CMOS manufacturing are both complex and rich; thus despite more than a decade of experience in CMOS manufacturing of nanoelectromechanical systems (NEMS) incorporating piezoelectrics, modern devices are still not meeting the optimal standards of performance. Several investigators cite reasons for performance deficiencies when comparing theoretical to experimental results ${ }^{6}$ but the one of particular interest is the result of delamination at the electrode/ piezoelectric interface ${ }^{7}$. Consequently the electric field essential to generate and sustain the piezoelelectric response of such devices is lost, resulting in device failure. Working on the hypothesis that buried conducting layers can both mitigate the delamination problem and generate sufficient electric field to engage the operation of energy scavenging devices, we have undertaken a study of silver ion implantation to experimentally assess its feasibility. The implanted sample is subsequently subjected to a thermal treatment to encourage diffusionassisted precipitation of the implanted species at high enough concentrations. The goal of this investigation is to explore the use of heavy ion implantation to develop a buried conducting layer in aluminum nitride that might be employed during device applications.

Multiple source reactive ion sputtering is used to deposit $\approx 1.8 \mu \mathrm{m}$ of AlN on a $525 \mu \mathrm{m}$ thick (100) Si substrate, followed by ion implantation into the aluminum nitride at $100 \mathrm{keV}$ to achieve a dose of $1.0 \times 10^{16} \mathrm{Ag}^{++} / \mathrm{cm}^{2}$ and subsequent annealing at temperatures between 400 and $1000^{\circ} \mathrm{C}$. Computer simulation models using Transport of Ions in Matter (TRIM) and TRIDYN 
are applied and confirmed by Rutherford Backscattering Spectrometry (RBS). X-ray diffraction and electron diffraction establish both the epitaxy of the AlN film on the (100) Si substrate and the crystalline quality of the epilayer prior to thermal annealing. Electron microscopy reveals that the sputtered AlN films grow epitaxially in a columnar morphology while electron diffraction (Figure 1 inset) confirmed both the epitaxy of the AlN film on the (001) Si substrate and the crystalline quality of the epilayer prior to the thermal annealing treatment. It is concluded that the Ag implantation does indeed have potential as a synthesis protocol for buried contact layer generation in piezoelectric based MEMS devices.

\section{References}

[1] H. Jaffe, J. American Ceramic Society 41 (1958) 494.

[2] B. Jaffe et al., J. Appl. Phys. 25 (6) (1954) 809.

[3] E. Cross, Nature 432 (2004) 24.

[4] T. Li et. al., in "III-V Semiconductors: Integration with Silicon-Based Microelectronics", ed. Li, T., Mastro, M., Dadgar, A. (CRC Press, Boca Raton) 593.

[5] X. Wang et. al., Journal of Micromechanics and Microengineering 13 (2003) 628.

[6] C.-M. Lin et. al., Adv. Mater. 24 (2012) 2722.

[7] F. Shanga et al., Int. J. Solids and Structures 46 (2004) 1729.

[8] The aid of Dr. T.-T. Yen, Dr. Debbie Senesky and Mr. Matt Ford of University of California, Berkeley and Dr. Andre Anders and Dr. Kin Man Yu of Lawrence Berkeley National Laboratory (LBL) is gratefully appreciated.

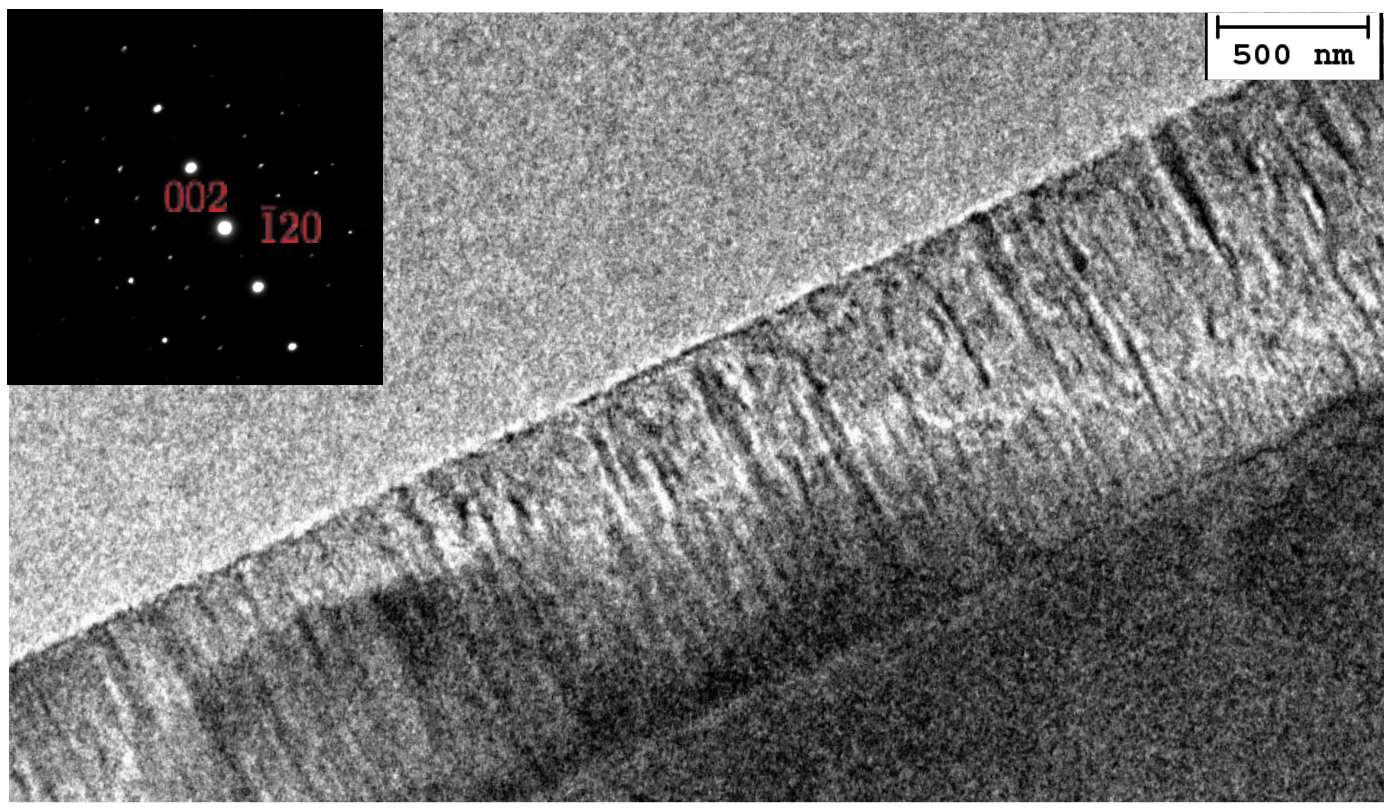

FIG. 1. Transmission electron micrograph of AIN columnar grain morphology after Ag ion implantation. The high crystalline quality and dominant [210] zone axis orientation of the columnar grains on (001) single crystalline Si substrate is demonstrated in the inset diffraction pattern. 